CUBO A Mathematical Journal

Vol.12, No-02, (29-42). June 2010

\title{
The method of Kantorovich majorants to nonlinear singular integral equations with Hilbert kernel
}

\author{
M. H. Saleh, S. M. Amer ${ }^{1}$ And M. H. Ahmed \\ Departement of Mathematics, \\ Faculty of Science, \\ Zagazig University, Zagazig, Egypt. \\ email: amrsammer@hotmail.com
}

\begin{abstract}
This paper concerned with applicability of the method of Kantorovich majorants to nonlinear singular integral equations with Hilbert kernel. The results are illustrated in Hölder space.

\section{RESUMEN}

Este artículo es concerniente a la aplicabilidad del método de mayorantes de Kantorovich para ecuaciones integrales singulares no lineales con núcleo de Hilbert. Los resultados son aplicaciones en espacios de Hölder.
\end{abstract}

Key words and phrases: Nonlinear singular integral equations, Kantorovich majorants method, Hölder spaces.

AMS 2000-Subject classification: $45 F 15,45 G 10$.

\section{Introduction}

There is a large literature on nonlinear singular integral equations with Hilbert and Cauchy kernel and related Riemann boundary value problems for analytic functions,cf.the monograph by Pogorzel-

\footnotetext{
${ }^{1}$ Corresponding author
} 
ski [16], Guseinov A.I. and Mukhtarov kh.sh. [9],Kantorovich L.V.[11],Muskhelishvill N.I.[14],and Mikhlin S.G.and Prossdorf S.[13]. The method of singular integral equations on closed contour has been intensively investigated by many approximation methods, specially method of modified NewtonKantorovich, reduction, collocation and mechanical quadratures, (see[1-6,10,12,15,17,19]).

In this paper the method of Kantorovich majorants[7,18,20], has been applied to the following class of nonlinear singular integral equations with Hilbert kernel :

$$
\varphi(t)=\lambda G\left(t, \frac{1}{2 \pi} \int_{0}^{2 \pi} g(t, \sigma, \varphi(\sigma)) \cot \frac{\sigma-t}{2} d \sigma\right),
$$

where $\lambda$ is a numerical parameter,

where

$$
v(t)=S g(t, \sigma, \varphi(\sigma))=\frac{1}{2 \pi} \int_{0}^{2 \pi} g(t, \sigma, \varphi(\sigma)) \cot \frac{\sigma-t}{2} d \sigma,
$$

then equation (1.1) takes the form:

$$
\varphi(t)=\lambda G(t, v(t))
$$

Now, we consider the equation:

$$
B(\varphi)=0,
$$

where

$$
(B \varphi)(t)=\varphi(t)-\lambda G(t, v(t)) .
$$

\section{Formulation of the problem}

Let $f: \bar{S}\left(\varphi_{0}, R\right) \subset X \longrightarrow Y$ be a nonlinear operator defined on the closure of a ball

$$
S\left(\varphi_{0}, R\right)=\left\{\varphi: \varphi \in X,\left\|\varphi-\varphi_{0}\right\|<R\right\}
$$

in a Banach space $\mathrm{X}$ into a Banach space $\mathrm{Y}$.

We give new conditions to ensure the convergence on Newton-Kantorovich approximations toward a solution of $f(\varphi)=0$, under the hypothesis that $\mathrm{f}$ is Frechet differentiable in $S\left(\varphi_{0}, R\right)$, and that it's derivative $\grave{f}$ satisfies the local Lipschitz condition :

$$
\left\|\grave{f}\left(\varphi_{1}\right)-\grave{f}\left(\varphi_{2}\right)\right\| \leq k(r)\left\|\varphi_{1}-\varphi_{2}\right\|, \varphi_{1}, \varphi_{2} \in \bar{S}\left(\varphi_{0}, r\right), o<r<R,
$$

where $k(r)$ is a non decreasing function on the interval $[0, R]$ and

$$
k(r)=\sup \left\{\frac{\left\|\grave{f}\left(\varphi_{1}\right)-\grave{f}\left(\varphi_{2}\right)\right\|}{\left\|\varphi_{1}-\varphi_{2}\right\|} \varphi_{1}, \varphi_{2} \in \bar{S}\left(\varphi_{0}, r\right) \varphi_{1} \neq \varphi_{2}\right\} .
$$

Define a scalar function $\psi:[0, R] \rightarrow(0, \infty)$ by

$$
\psi(r)=a+b \int_{0}^{r} w(t) d t-r,
$$


using the function

$$
w(r)=\int_{0}^{r} k(t) d t
$$

and

$$
a=\left\|\grave{f}\left(\varphi_{0}\right)^{-1} f\left(\varphi_{0}\right)\right\|, \quad b=\left\|\grave{f}\left(\varphi_{0}\right)^{-1}\right\| .
$$

Theorem $2.1[4,7]$ Suppose that the equation (2.3)has a unique positive root $r_{*}$ in $[0, R]$ and $\psi(R) \leq 0$. Then the equation $f(\varphi)=0$ has a unique solution $\varphi_{*}$ in $S\left(\varphi_{0}, R\right)$ and the NewtonKantorovich approximations:

$$
\varphi_{n}=\varphi_{n-1}-\grave{f}\left(\varphi_{n-1}\right)^{-1} f\left(\varphi_{n-1}\right), \quad n \in N,
$$

are defined for all $n \in N$, belong to $S\left(\varphi_{0}, r_{*}\right)$ and converges to $\varphi_{*}$.

Moreover, the following estimate holds

$$
\left\|\varphi_{n+1}-\varphi_{n}\right\| \leq r_{n+1}-r_{n}, \quad\left\|\varphi_{*}-\varphi_{n}\right\| \leq r_{*}-r_{n},
$$

where the sequence $\left(r_{n}\right)_{n \in N}$ converges to $r_{*}$, is defined by the recurrence formula

$$
r_{0}=0, \quad r_{n+1}=r_{n}-\frac{\psi\left(r_{n}\right)}{\psi\left(\grave{r}_{n}\right)}, \quad n \in N
$$

In the present paper, we investigate some sufficient conditions, which ensure that the class of nonlinear singular integral equations (1.1) verifies the hypotheses of theorem (2.1).

\section{Some auxiliary results}

Definition 3.1[9] We denote by $H_{\delta}, 0<\delta<1$,the Hölder space of continuous functions, which satisfy the Hölder condition with exponent $\delta$ with norm

$$
\|\varphi\|_{\delta}=\|\varphi\|_{c}+H^{\delta}(\varphi)
$$

where

$$
\|\varphi\|_{c}=\max _{\sigma \in[0,2 \pi]}|\varphi(\sigma)|
$$

and

$$
H^{\delta}(\varphi)=\sup _{\sigma_{1} \neq \sigma_{2}} \frac{\left|\varphi\left(\sigma_{1}\right)-\varphi\left(\sigma_{2}\right)\right|}{\left|\sigma_{1}-\sigma_{2}\right|^{\delta}}
$$

Lemma 3.1 [9] Let the functions $G(t, v(t)), g(t, \sigma, \varphi(\sigma))$ and it's partial derivatives up to second order, satisfy the following conditions

$$
\left|\frac{\partial^{m} G\left(t_{1}, v\left(t_{1}\right)\right)}{\partial v^{m}}-\frac{\partial^{m} G\left(t_{2}, v\left(t_{2}\right)\right)}{\partial v^{m}}\right| \leq c_{m}(r)\left\{\left|t_{1}-t_{2}\right|^{\delta}+\left|v\left(t_{1}\right)-v\left(t_{2}\right)\right|\right\},
$$


and

$$
\left|\frac{\partial^{m} g_{\varphi}\left(t_{1}, \sigma_{1}, \varphi\left(\sigma_{1}\right)\right)}{\partial \varphi^{m}}-\frac{\partial^{m} g_{\varphi}\left(t_{2}, \sigma_{2}, \varphi\left(\sigma_{2}\right)\right)}{\partial \varphi^{m}}\right| \leq a_{m}(r)\left\{\left|t_{1}-t_{2}\right|^{\delta}+\left|\sigma_{1}-\sigma_{2}\right|^{\delta}+\left|\varphi\left(t_{1}\right)-\varphi\left(t_{2}\right)\right|\right\}
$$

where $c_{m}(r), a_{m}(r)$ are positive increasing functions $\mathrm{m}=0,1,2$ and $t_{i}, \sigma_{i} \in[0,2 \pi], i=1,2$. If $\varphi(\sigma) \in H_{\delta}$, then $G(t, v(t)), g(t, \sigma, \varphi(\sigma)) \in H_{\delta}$.

Lemma 3.2 If the functions $G(t, v(t))$ and $g(t, \sigma, \varphi(\sigma))$ satisfy the conditions of lemma(3.1), then the operator $B(\varphi)$ has a Frechet derivative at every fixed point in the space $H_{\delta}$ and its derivative is given by

$$
\grave{B}(\varphi) h=h(t)-\lambda G_{v}(t, v(t)) S g_{\varphi}(t, \sigma, \varphi(\sigma)) h(\sigma) .
$$

Moreover it satisfies Lipschitz condition:

$$
\left\|\grave{B}\left(\varphi_{1}\right)-\grave{B}\left(\varphi_{2}\right)\right\| \leq k(r)\left\|\varphi_{1}-\varphi_{2}\right\|,
$$

for all $\varphi_{1}, \varphi_{2} \in S\left(\varphi_{0}, r\right)$ and $o<r<R$.

Proof Let $\varphi(t)$ be any fixed point in the space $0,<\delta<1$ and $h(t)$ be any arbitrary element in $H_{\delta}$ , then we obtain :

$$
\begin{aligned}
B(\varphi+h)-B(\varphi) & =h(t)-\lambda[G(t, S g(t, \sigma, \varphi(\sigma)+h(\sigma)))-G(t, S g(t, \sigma, \varphi(\sigma)))] \\
& =\grave{B}(\varphi) h+\eta(t, h),
\end{aligned}
$$

where $0 \leq \xi \leq 1$ and

$$
\begin{aligned}
\eta(t, h) & =\lambda \int_{0}^{1}(1-\xi)\left[G_{v^{2}}(t, S g(t, \sigma, \varphi(\sigma)+\xi h(\sigma)))\left(S g_{\varphi}(t, \sigma, \varphi(\sigma)+\xi h(\sigma)) h(\sigma)\right)^{2}\right. \\
& \left.+G_{v}(t, S g(t, \sigma, \varphi(\sigma)+\xi h(\sigma))) S g_{\varphi^{2}}(t, \sigma, \varphi(\sigma)+\xi h(\sigma)) h(\sigma)^{2}\right] d \xi .
\end{aligned}
$$

Now, we shall prove that

$$
\lim _{\|h\| \rightarrow 0} \frac{\|\eta(t, h)\|}{\|h\|}=0
$$

Using the inequalities $[9,13]$

$$
\left.\begin{array}{l}
\left\|\int_{a}^{b} \frac{y(s)}{s-x} d s\right\| \leq \rho_{0}\|y\| \text {, where } \rho_{0} \text { is a positive constant } \\
\|u v\| \leq\|u\|\|v\| \text { for all } u, v \in H_{\delta}
\end{array}\right\} \text {. }
$$

Now;

$$
\begin{aligned}
\|\eta(t, h)\| & \leq\left\|h(\sigma)^{2}\right\| \rho_{0}\left[\left\|G_{v^{2}}(t, S g(t, \sigma, \varphi(\sigma)))\right\|\left\|\left(g_{\varphi}(t, \sigma, \varphi(\sigma))\right)^{2}\right\|\right. \\
& \left.+\left\|G_{v}(t, S g(t, \sigma, \varphi(\sigma)))\right\|\left\|g_{\varphi^{2}}(t, \sigma, \varphi(\sigma))\right\|\right] .
\end{aligned}
$$

Hence

$$
\lim _{\|h\| \rightarrow 0} \frac{\|\eta(t, h)\|}{\|h\|}=0
$$


which prove the differentiability of $B(\varphi)$ in the sense of Frechet and its derivative is given by (3.4).

To prove the Frechet derivative $\grave{B}(\varphi)$ satisfies Lipschitz condition in the sphere

$$
S\left(\varphi_{0}, R\right)=\left\{\varphi:\left\|\varphi-\varphi_{0}\right\|<R\right\} .
$$

We consider

$$
\begin{aligned}
\left\|\grave{B}\left(\varphi_{1}\right) h-\grave{B}\left(\varphi_{2}\right) h\right\| & =\| \lambda G_{v}\left(t, S g\left(t, \sigma, \varphi_{1}(\sigma)\right)\right) S g_{\varphi}\left(t, \sigma, \varphi_{1}(\sigma)\right) h(\sigma) \\
& -\lambda G_{v}\left(t, S g\left(t, \sigma, \varphi_{2}(\sigma)\right)\right) S g_{\varphi}\left(t, \sigma, \varphi_{2}(\sigma)\right) h(\sigma) \| \\
& \leq \mid \lambda\|h\|\left[\left\|G_{v}\left(t, v_{1}(t)\right)\right\|\left\|S g_{\varphi}\left(t, \sigma, \varphi_{1}(\sigma)\right)-S g_{\varphi}\left(t, \sigma, \varphi_{2}(\sigma)\right)\right\|\right. \\
& \left.+\left\|S g_{\varphi}\left(t, \sigma, \varphi_{2}(\sigma)\right)\right\|\left\|G_{v}\left(t, v_{1}(t)\right)-G_{v}\left(t, v_{2}(t)\right)\right\|\right] \\
& \leq\|h\| k(r)\left\|\varphi_{1}-\varphi_{2}\right\|,
\end{aligned}
$$

where $k(r)=|\lambda| \rho_{0}\left[a_{1}(r) D+\left\|g_{\varphi}\right\| c_{1}(r) a_{0}(r)\right]$, and $D=\max _{t}\left|G_{v}(t, S g(t, \sigma, \varphi(\sigma)))\right|$ then the lemma is proved.

\section{Solution of linear singular integral equation}

To find the operator $\grave{B}\left(\varphi_{0}\right)^{-1}$, we investigate the solution of the equation

$$
h(t)-\frac{\lambda G_{v}(t, v(t))}{2 \pi} \int_{0}^{2 \pi} g_{\varphi}(t, \sigma, \varphi(\sigma)) h(\sigma) \cot \frac{\sigma-t}{2} d \sigma=f(t) .
$$

For this aim we introduce the following theorem:

Theorem 4.1 If the functions $G(t, v(t))$ and $g(t, \sigma, \varphi(\sigma))$ satisfy the conditions of lemma(3.2), then the linear operator defined by (3.4) has a bounded inverse $\grave{B}\left(\varphi_{0}\right)^{-1}$ for any fixed $\varphi_{0} \in H_{\delta},(0<\delta<1)$.

\section{Proof}

Let us transform the equation (4.1) by introducing new variables :

$$
s=e^{i t}, \tau=e^{i \sigma}, d \tau=i e^{i \sigma} d \sigma,
$$

since

$$
\frac{1}{2} \cot \frac{\sigma-t}{2} d \sigma=\left(\frac{1}{\tau-s}-\frac{1}{2 \tau}\right) d \tau,
$$

then equation (4.1) has the form

$$
h(s)-\frac{\lambda X_{v}(s, v(s))}{\pi i} \int_{\gamma} i k_{\varphi}(s, \tau, \varphi(\tau)) h(\tau)\left(\frac{1}{\tau-s}-\frac{1}{2 \tau}\right) d \tau=f(s),
$$

where $\gamma$ is a unit circle, $G_{v}(t, v(t))=X_{v}(s, v(s))$ and $g_{\varphi}(t, \sigma, \varphi(\sigma))=k_{\varphi}(s, \tau, \varphi(\tau))$.

We introduce the sectionally holomorphic function of variable $z$ as follows:

$$
H(z)=\frac{\lambda X_{v}(s, v(s))}{2 \pi i} \int_{\gamma} \frac{i k_{\varphi}(s, \tau, \varphi(\tau))}{\tau-z} h(\tau) d \tau-C,
$$


and

$$
\begin{aligned}
H(\infty) & =-C=\frac{-\lambda X_{v}(s, v(s))}{4 \pi} \int_{\gamma} \frac{i k_{\varphi}(s, \tau, \varphi(\tau))}{\tau} h(\tau) d \tau \\
& =\frac{-i \lambda G_{v}(t, v(t))}{4 \pi} \int_{0}^{2 \pi} g_{\varphi}(t, \sigma, \varphi(\sigma)) h(\sigma) d \sigma .
\end{aligned}
$$

According to Sokhotoski formulae[9], we have

$$
\begin{aligned}
H^{ \pm}(s) & = \pm \frac{i \lambda X_{v}(s, v(s))}{2} k_{\varphi}(s, s, \varphi(s)) h(s) \\
& +\frac{\lambda X_{v}(s, v(s))}{2 \pi i} \int_{\gamma} \frac{i k_{\varphi}(s, \tau, \varphi(\tau))}{\tau-s} h(\tau) d \tau-C .
\end{aligned}
$$

Therefore

$$
\left.\begin{array}{c}
H^{+}(s)-H^{-}(s)=i \lambda X_{v}(s, v(s)) k_{\varphi}(s, s, \varphi(s)) h(s) \\
H^{+}(s)+H^{-}(s)=\frac{\lambda X_{v}(s, v(s))}{\pi i} \int_{\gamma} \frac{i k_{\varphi}(s, \tau, \varphi(\tau))}{\tau-s} h(\tau) d \tau-2 C
\end{array}\right\} .
$$

Substituting from equation (4.4) into equation (4.2)we have

$$
h(s)-f(s)+2 C=H^{+}(s)+H^{-}(s)+2 C .
$$

Hence we get

$$
h(s)=H^{+}(s)+H^{-}(s)+f(s),
$$

therefore from (4.4) and (4.6) we have,

$$
h(s)\left[1 \pm i \lambda X_{v}(s, v(s)) k_{\varphi}(s, s, \varphi(s))\right]=2 H^{ \pm}(s)+f(s),
$$

since $1 \pm i \lambda X_{v}(s, v(s)) k_{\varphi}(s, s, \varphi(s)) \neq 0$, then the last conditions equivalent to the following

$$
\left.\begin{array}{l}
h(s)=\frac{2 H^{+}(s)}{1+i \lambda X_{v}(s, v(s)) k_{\varphi}(s, s, \varphi(s))}+\frac{f(s)}{1+i \lambda X_{v}(s, v(s)) k_{\varphi}(s, s, \varphi(s))}, \\
h(s)=\frac{2 H^{-}(s)}{1-i \lambda X_{v}(s, v(s)) k_{\varphi}(s, s, \varphi(s))}+\frac{f(s)}{1-i \lambda X_{v}(s, v(s)) k_{\varphi}(s, s, \varphi(s))}
\end{array}\right\} .
$$

By equating the right hand side of equation (4.7) we get the Riemann boundary value problem

$$
H^{+}(s)=\frac{1+i \lambda X_{v}(s, v(s)) k_{\varphi}(s, s, \varphi(s))}{1-i \lambda X_{v}(s, v(s)) k_{\varphi}(s, s, \varphi(s))} H^{-}(s)+\frac{i \lambda X_{v}(s, v(s)) k_{\varphi}(s, s, \varphi(s))}{1-i \lambda X_{v}(s, v(s)) k_{\varphi}(s, s, \varphi(s))} f(s) .
$$

It is well known that the index of equation (4.8) is zero[8],then

$$
\frac{1+i \lambda X_{v}(s, v(s)) k_{\varphi}(s, s, \varphi(s))}{1-i \lambda X_{v}(s, v(s)) k_{\varphi}(s, s, \varphi(s))}=\frac{X^{+}(s)}{X^{-}(s)}
$$

where

$$
X(z)=\frac{1}{2 \pi} \int_{\gamma} \ln \frac{1+i \lambda X_{v}(s, v(s)) i k_{\varphi}(s, \tau, \varphi(\tau))}{1-i \lambda X_{v}(s, v(s)) k_{\varphi}(s, \tau, \varphi(\tau))} \frac{d \tau}{\tau-z}
$$


the problem (4.8)can be written in the form

$$
\frac{H^{+}(s)}{X^{+}(s)}-\frac{H^{-}(s)}{X^{-}(s)}=\frac{i \lambda X_{v}(s, v(s)) k_{\varphi}(s, s, \varphi(s)) f(s)}{1-i \lambda X_{v}(s, v(s)) k_{\varphi}(s, s, \varphi(s)) X^{+}(s)}
$$

Hence ,from [8], the boundary value problem (4.8) has the solution

$$
H(z)=X(z)\left[\frac{\lambda X_{v}(s, v(s))}{2 \pi i} \int_{\gamma} \frac{i k_{\varphi}(s, \tau, \varphi(\tau)) f(\tau)}{X^{+}(\tau)\left(1-i \lambda X_{v}(s, v(s)) k_{\varphi}(s, \tau, \varphi(\tau))\right)} \frac{d \tau}{\tau-s}-C\right] .
$$

By Sokhotski formulae

$$
\begin{aligned}
H^{+}(s) & =\frac{i \lambda X_{v}(s, v(s)) k_{\varphi}(s, s, \varphi(s)) f(s)}{2\left(1-i \lambda X_{v}(s, v(s)) k_{\varphi}(s, s, \varphi(s))\right)} \\
& +\frac{\lambda X_{v}(s, v(s)) X^{+}(s)}{2 \pi i} \int_{\gamma} \frac{i k_{\varphi}(s, \tau, \varphi(\tau)) f(\tau)}{X^{+}(\tau)\left(1-i \lambda X_{v}(s, v(s)) k_{\varphi}(s, \tau, \varphi(\tau))\right)} \frac{d \tau}{\tau-s} \\
& -C X^{+}(s) .
\end{aligned}
$$

Substituting from (4.9) into (4.7) we have,

$$
\begin{aligned}
h(s) & =\frac{f(s)}{u(s)}+\frac{z(s) \lambda X_{v}(s, v(s))}{u(s) \pi i} \int_{\gamma} \frac{i k_{\varphi}(s, \tau, \varphi(\tau)) f(\tau)}{z(\tau)} \frac{d \tau}{\tau-s} \\
& -\frac{2 C z(s)}{u(s)}
\end{aligned}
$$

where

$$
\begin{gathered}
u(s)=1+\lambda^{2} X_{v}^{2}(s, v(s)) k_{\varphi}^{2}(s, s, \varphi(s)), \\
z(s)=\sqrt{u(s)} e^{\Gamma(s)},
\end{gathered}
$$

and

$$
\Gamma(s)=\frac{1}{2 \pi i} \int_{\gamma} \ln \frac{1+i \lambda X_{v}(s, v(s)) i k_{\varphi}(s, \tau, \varphi(\tau))}{1-i \lambda X_{v}(s, v(s)) k_{\varphi}(s, \tau, \varphi(\tau))} \frac{d \tau}{\tau-s},
$$

since

$$
\frac{d \tau}{\tau-s}=\frac{1}{2} \cot \frac{\sigma-t}{2}+\frac{i}{2} d \sigma .
$$

Hence

$$
\begin{aligned}
z\left(e^{i t}\right)=z(s) & =\sqrt{u(t)} \exp \left(\frac{1}{4 \pi} \int_{0}^{2 \pi} \ln \frac{1+i \lambda G_{v}(t, v(t)) g_{\varphi}(t, \sigma, \varphi(\sigma))}{1-i \lambda G_{v}(t, v(t)) g_{\varphi}(t, \sigma, \varphi(\sigma))} d \sigma\right. \\
& \left.\exp \quad\left(\frac{1}{4 \pi i} \int_{0}^{2 \pi} \ln \frac{1+i \lambda G_{v}(t, v(t)) g_{\varphi}(t, \sigma, \varphi(\sigma))}{1-i \lambda G_{v}(t, v(t)) g_{\varphi}(t, \sigma, \varphi(\sigma))} \cot \frac{\sigma-t}{2} d \sigma\right)\right) .
\end{aligned}
$$


Now we determine the constant $C$ as follows

$$
\begin{aligned}
C & =\frac{i \lambda G_{v}(t, v(t))}{4 \pi} \int_{0}^{2 \pi} g_{\varphi}(t, \sigma, \varphi(\sigma)) h(\sigma) d \sigma= \\
& =\left(1+\frac{i z(t) \lambda G_{v}(t, v(t))}{2 \pi u(t)} \int_{0}^{2 \pi} g_{\varphi}(t, \sigma, \varphi(\sigma)) d \sigma\right)^{-1} \\
& {\left[\frac { i \lambda G _ { v } ( t , v ( t ) ) } { 4 \pi } \int _ { 0 } ^ { 2 \pi } g _ { \varphi } ( t , \sigma , \varphi ( \sigma ) ) \left[\frac{f(t)}{u(t)}\right.\right.} \\
& +\frac{z(t)}{2 \pi u(t)} \int_{0}^{2 \pi} \frac{g_{\varphi}(\xi, \sigma, \varphi(\sigma)) f(\xi)}{z(\xi)} \cot \frac{\xi-\sigma}{2} d \xi \\
& \left.\left.+\frac{i z(t)}{2 \pi u(t)} \int_{0}^{2 \pi} \frac{\lambda G_{v}(\xi, v(\xi)) g_{\varphi}(\xi, \sigma, \varphi(\sigma)) f(\xi)}{z(\xi)} d \xi\right] d \sigma .\right\}
\end{aligned}
$$

Then

$$
\begin{aligned}
h(t) & =\frac{f(t)}{u(t)}+\frac{\lambda G_{v}(t, v(t)) z(t)}{2 \pi u(t)} \int_{0}^{2 \pi} \frac{g_{\varphi}(t, \sigma, \varphi(\sigma)) f(\sigma)}{z(\sigma)} \cot \frac{\sigma-t}{2} d \sigma \\
& +\frac{\lambda G_{v}(t, v(t)) z(t)}{2 \pi u(t)} \int_{0}^{2 \pi} \frac{g_{\varphi}(t, \sigma, \varphi(\sigma)) f(\sigma)}{z(\sigma)} d \sigma-\frac{2 C z(t)}{u(t)} \\
& =\grave{B}\left(\varphi_{0}\right)^{-1} f(t) .
\end{aligned}
$$

We shall prove that the operator $\grave{B}\left(\varphi_{0}\right)^{-1}$ is bounded.

It is easy to prove that $v(t), \Gamma(t)$ and $z(t) \in H_{\delta}$ therefore by using inequality (3.6) we get

$$
\begin{aligned}
\left\|\grave{B}\left(\varphi_{0}\right)^{-1}\right\|_{\delta} & \leq\left\|\frac{1}{u}\right\|_{\delta}\left\{1+\rho_{0}|\lambda|\|z\|_{\delta}\left\|G_{v}(t, v(t))\right\|_{\delta}\left\|g_{\varphi}(t, t, \varphi(t))\right\|_{\delta}\left\|\frac{1}{z}\right\|_{\delta}\right. \\
& \left.+\rho_{1}|\lambda|\|z\|_{\delta}\left\|G_{v}(t, v(t))\right\|_{\delta}+2 \tilde{C}\|z\|_{\delta}\right\}
\end{aligned}
$$

where

$$
\rho_{1}=\frac{1}{2 \pi} \int_{0}^{2 \pi}\left|\frac{g_{\varphi}(t, \sigma, \varphi(\sigma))}{z(\sigma)}\right| d \sigma
$$

and

$$
\begin{aligned}
\tilde{C} & =\left(1+\frac{i z(t) \lambda G_{v}(t, v(t))}{2 \pi u(t)} \int_{0}^{2 \pi} g_{\varphi}(t, \sigma, \varphi(\sigma)) d \sigma\right)^{-1} \\
& {\left[\frac { i \lambda G _ { v } ( t , v ( t ) ) } { 4 \pi } \int _ { 0 } ^ { 2 \pi } g _ { \varphi } ( t , \sigma , \varphi ( \sigma ) ) \left[\frac{1}{u(t)}\right.\right.} \\
& +\frac{z(t)}{2 \pi u(t)} \int_{0}^{2 \pi} \frac{g_{\varphi}(\xi, \sigma, \varphi(\sigma))}{z(\xi)} \cot \frac{\xi-\sigma}{2} d \xi \\
& \left.\left.+\frac{i z(t)}{2 \pi u(t)} \int_{0}^{2 \pi} \frac{\lambda G_{v}(\xi, v(\xi)) g_{\varphi}(\xi, \sigma, \varphi(\sigma))}{z(\xi)} d \xi\right]\right] d \sigma .
\end{aligned}
$$

We determine the norm of each term in right hand side of the above inequality. From definition (3.1) we have

$$
\left\|\frac{1}{u}\right\|_{c}=\left\|\frac{1}{1+\lambda^{2} G_{v}^{2}(t, v(t)) g_{\varphi}^{2}(t, t, \varphi(t))}\right\|_{c} \leq 1
$$




$$
\begin{aligned}
\left|\frac{1}{u\left(t_{1}\right)}-\frac{1}{u\left(t_{2}\right)}\right| & \leq\left|u\left(t_{1}\right)-u\left(t_{2}\right)\right| \leq \mid \lambda^{2} \| G_{v}^{2}\left(t_{1}, v\left(t_{1}\right)\right) g_{\varphi}^{2}\left(t_{1}, t_{1}, \varphi\left(t_{1}\right)\right) \\
& -G_{v}^{2}\left(t_{2}, v\left(t_{2}\right)\right) g_{\varphi}^{2}\left(t_{2}, t_{2}, \varphi\left(t_{2}\right)\right) \mid \\
\leq & \lambda^{2}\left[\left|G_{v}\left(t_{1}, v\left(t_{1}\right)\right) g_{\varphi}\left(t_{1}, t_{1}, \varphi\left(t_{1}\right)\right)-G_{v}\left(t_{2}, v\left(t_{2}\right)\right) g_{\varphi}\left(t_{2}, t_{2}, \varphi\left(t_{2}\right)\right)\right|\right] \\
& {\left[2\left\|G_{v}(t, v(t))\right\|_{c}\left\|g_{\varphi}(t, t, \varphi(t))\right\|_{c}\right], }
\end{aligned}
$$

since

$$
\left\|G_{v}(t, v(t))\right\|_{c} \leq c_{1}(r)\|v\|_{c}+\left\|G_{v}(t, 0)\right\|_{c}
$$

similarly

$$
\left\|g_{\varphi}(t, t, \varphi(t))\right\|_{c} \leq a_{1}(r)\|\varphi\|_{c}+\left\|g_{\varphi}(t, t, 0)\right\|_{c},
$$

using conditions(3.2)and (3.3)we have

$$
\begin{gathered}
\left|g_{\varphi}\left(t_{1}, t_{1}, \varphi\left(t_{1}\right)\right)-g_{\varphi}\left(t_{2}, t_{2}, \varphi\left(t_{2}\right)\right)\right| \leq a_{1}(r)\left(2+H^{\delta}(\varphi)\right)\left|t_{1}-t_{2}\right|^{\delta}, \\
\left.\quad\left|G_{v}\left(t_{1}, v\left(t_{1}\right)\right)-G_{v}\left(t_{2}, v\left(t_{2}\right)\right)\right| \leq c_{1}(r)\left(1+H^{\delta}(v)\right)\right)\left|t_{1}-t_{2}\right|^{\delta} .
\end{gathered}
$$

and

$$
\left.\left|G_{v}\left(t_{2}, v\left(t_{2}\right)\right)\right| \leq \mid G_{v}\left(t_{2}, 0\right)\right) \mid+c_{1}(r)\left(\left|v\left(t_{2}\right)\right|\right)
$$

similarly

$$
\left|g_{\varphi}\left(t_{1}, t_{1}, \varphi\left(t_{1}\right)\right)\right| \leq a_{1}(r)|\varphi|+\left|g_{\varphi}\left(t_{1}, t_{1}, 0\right)\right| .
$$

Hence

$$
\left|\frac{1}{u\left(t_{1}\right)}-\frac{1}{u\left(t_{2}\right)}\right| \leq \lambda^{2} \beta
$$

So

$$
\left\|\frac{1}{u}\right\|_{\delta} \leq R_{1}
$$

where $R_{1}=1+\lambda^{2} \beta$ and

$$
\begin{aligned}
\beta & =\left[\left(\left|g_{\varphi}\left(t_{1}, t_{1}, 0\right)\right|+a_{1}(r)|\varphi|\right)\left(c_{1}(r)\left(1+H^{\delta}(v)\right)\left|t_{1}-t_{2}\right|^{\delta}\right)\right. \\
& \left.+\left(\left|G_{v}\left(t_{2}, 0\right)\right|+c_{1}(r)|v|\right)\left(a_{1}(r)\left(2+H^{\delta}(\varphi)\right)\left|t_{1}-t_{2}\right|^{\delta}\right)\right] \\
& {\left[\quad\left(c_{1}(r)\|v\|_{c}+\left\|G_{v}(t, 0)\right\|_{c}\right)\left(a_{1}(r)\|\varphi\|_{c}+\left\|g_{\varphi}(t, t, 0)\right\|_{c}\right)\right], }
\end{aligned}
$$

To determine $\|z\|_{\delta}$ we get

$$
\|z\|_{\delta} \leq\|\sqrt{u}\|_{\delta}\left(1+\|\Gamma\|_{\delta}\right) e^{\|\Gamma\|_{\delta}}
$$

since

$$
\|u\|_{c} \leq \sqrt{1+\lambda^{2}\left(c_{1}\|v\|_{c}+\left\|G_{v}(t, 0)\right\|_{c}\right)^{2}\left(a_{1}\|\varphi\|_{c}+\left\|g_{\varphi}(t, t, 0)\right\|_{c}\right)^{2}} .
$$

By 
applying Lagrange theorem:

$$
\begin{aligned}
\left|\sqrt{u\left(t_{1}\right)}-\sqrt{u\left(t_{2}\right)}\right| & =\mid \frac{1}{2}(1+\theta)^{-1 / 2} \lambda^{2}\left[G_{v}^{2}\left(t_{1}, v\left(t_{1}\right)\right) g_{\varphi}^{2}\left(t_{1}, t_{1}, \varphi\left(t_{1}\right)\right)\right. \\
& \left.-G_{v}^{2}\left(t_{2}, v\left(t_{2}\right)\right) g_{\varphi}^{2}\left(t_{2}, t_{2}, \varphi\left(t_{2}\right)\right)\right] \mid \\
& \leq \lambda^{2} \beta
\end{aligned}
$$

where $\theta$ between $\lambda G_{v}\left(t_{1}, v\left(t_{1}\right)\right) g_{\varphi}\left(t_{1}, t_{1}, \varphi\left(t_{1}\right)\right)$ and $\lambda G_{v}\left(t_{2}, v\left(t_{2}\right)\right) g_{\varphi}\left(t_{2}, t_{2}, \varphi\left(t_{2}\right)\right)$.

Then

$$
\|\sqrt{u}\|_{\delta} \leq R_{2}
$$

where

$$
R_{2}=\sqrt{1+\left(c_{1}\|v\|_{c}+\left\|G_{v}(t, 0)\right\|_{c}\right)^{2}\left(a_{1}\|\varphi\|_{c}+\left\|g_{\varphi}(t, t, 0)\right\|_{c}\right)^{2}}+\lambda^{2} \beta .
$$

Also, we determine $\|\Gamma\|_{\delta}$, since

$$
\Gamma(t)=\frac{1}{2 \pi} \int_{0}^{2 \pi} \operatorname{arctg} \lambda G_{v}(t, v(t)) g_{\varphi}(t, \sigma, \varphi(\sigma)) \cot \frac{\sigma-t}{2} d \sigma+Q,
$$

where

$$
Q=\frac{1}{4 \pi} \int_{0}^{2 \pi} \ln \frac{1+i \lambda G_{v}(t, v(t)) g_{\varphi}(t, \sigma, \varphi(\sigma))}{1-i \lambda G_{v}(t, v(t)) g_{\varphi}(t, \sigma, \varphi(\sigma))} d \sigma
$$

by using (3.6)we have

$$
\begin{aligned}
&\|\Gamma\|_{c} \leq \rho_{0}\left\|\operatorname{arctg} \lambda G_{v}(t, v(t)) g_{\varphi}(t, t, \varphi(t))\right\|_{c}+|Q| \leq \frac{\rho_{0} \pi}{2}+|Q|, \\
&\left|\operatorname{arctg} \lambda G_{v}\left(t_{1}, v\left(t_{1}\right)\right) g_{\varphi}\left(t_{1}, t_{1}, \varphi\left(t_{1}\right)\right)-\operatorname{arctg} \lambda G_{v}\left(t_{2}, v\left(t_{2}\right)\right) g_{\varphi}\left(t_{2}, t_{2}, \varphi\left(t_{2}\right)\right)\right| \\
& \leq\left|\frac{\lambda}{1+\theta_{1}^{2}}\left[G_{v}\left(t_{1}, v\left(t_{1}\right)\right) g_{\varphi}\left(t_{1}, t_{1}, \varphi\left(t_{1}\right)\right)-G_{v}\left(t_{2}, v\left(t_{2}\right)\right) g_{\varphi}\left(t_{2}, t_{2}, \varphi\left(t_{2}\right)\right)\right]\right| \\
& \leq|\lambda|\left[\left(\left|g_{\varphi}\left(t_{1}, t_{1}, 0\right)\right|+a_{1}(r)|\varphi|\right)\left(c_{1}(r)\left(1+H^{\delta}(v)\right)\left|t_{1}-t_{2}\right|^{\delta}\right)\right. \\
&\left.\quad+\left(\left|G_{v}\left(t_{2}, 0\right)\right|+c_{1}(r)|v|\right)\left(a_{1}(r)\left(2+H^{\delta}(\varphi)\right)\left|t_{1}-t_{2}\right|^{\delta}\right)\right],
\end{aligned}
$$

where $\theta_{1}$ between $\lambda G_{v}\left(t_{1}, v\left(t_{1}\right)\right) g_{\varphi}\left(t_{1}, t_{1}, \varphi\left(t_{1}\right)\right)$ and $\lambda G_{v}\left(t_{2}, v\left(t_{2}\right)\right) g_{\varphi}\left(t_{2}, t_{2}, \varphi\left(t_{2}\right)\right)$. Therefore

$$
\|\Gamma\|_{\delta} \leq R_{3}
$$

where

$$
\begin{aligned}
R_{3} & =\frac{\rho_{0} \pi}{2}+|Q|+|\lambda|\left[\left(\left|g_{\varphi}\left(t_{1}, t_{1}, 0\right)\right|\right.\right. \\
& +a_{1}(r)|\varphi|\left(c_{1}(r)\left(1+H^{\delta}(v)\right)\left|t_{1}-t_{2}\right|^{\delta}\right) \\
& \left.+\left(\left|G_{v}\left(t_{2}, 0\right)\right|+c_{1}(r)|v|\right)\left(a_{1}(r)\left(2+H^{\delta}(\varphi)\right)\left|t_{1}-t_{2}\right|^{\delta}\right)\right] .
\end{aligned}
$$

Substituting from (4.14) and (4.15) into (4.13) we have

$$
\|z\|_{\delta} \leq R_{2}\left(1+R_{3}\right) e^{R_{3}} .
$$


From (4.14) we can determine $\left\|\frac{1}{z}\right\|_{\delta}$,

$$
\left\|\frac{1}{z}\right\|_{\delta} \leq \frac{1}{\|\sqrt{u}\|_{\delta}}\left(1+\|\Gamma\|_{\delta}\right) e^{\|\Gamma\|_{\delta}} .
$$

But

$$
\left\|\frac{1}{\sqrt{u}}\right\|_{c} \leq\left\|\frac{1}{\sqrt{1+\lambda^{2} G_{v}^{2}\left(t_{2}, v\left(t_{2}\right) g_{\varphi}^{2}\left(t_{2}, t_{2}, \varphi\left(t_{2}\right)\right)\right.}}\right\|_{c} \leq 1
$$

and

$$
\left|\frac{1}{\sqrt{u\left(t_{1}\right)}}-\frac{1}{\sqrt{u\left(t_{2}\right)}}\right| \leq\left|\sqrt{u\left(t_{1}\right)}-\sqrt{u\left(t_{2}\right)}\right| \leq \lambda^{2} \beta
$$

then

$$
\left\|\frac{1}{\sqrt{u}}\right\|_{\delta} \leq R_{4},
$$

where

$$
R_{4}=\left(1+\lambda^{2} \beta\right) .
$$

So that

$$
\left\|\frac{1}{z}\right\|_{\delta} \leq R_{4}\left(1+R_{3}\right) e^{R_{3}} .
$$

Then:

$$
\left\|\grave{B}\left(\varphi_{0}\right)^{-1}\right\| \leq \alpha_{0}
$$

where

$$
\begin{aligned}
\alpha_{0} & =R_{1}\left(1+\rho|\lambda| R_{2}\left(1+R_{3}\right) e^{R_{3}}\right)\left(\left\|G_{v}(t, 0)\right\|_{c}\right. \\
& +c_{1}(r)(1+\|v\|)\left(a_{1}(r)(2+\|\varphi\|)\right. \\
& \left.+\left\|g_{\varphi}(t, t, 0)\right\|_{c}\right)\left(R_{4}\left(1+R_{3}\right) e^{R_{3}}\right) \\
& \left.+\left|\rho_{1} \| \lambda\right| R_{2}\left(1+R_{3}\right) e^{R_{3}}\right)\left(\left\|G_{v}(t, 0)\right\|_{c}+c_{1}(r)(1+\|v\|)\right. \\
& +2 \tilde{C} R_{2}\left(1+R_{3}\right) e^{R_{3}},
\end{aligned}
$$

Hence the theorem is proved.

Now, we determine $\left\|\grave{B}\left(\varphi_{0}\right)^{-1} B\left(\varphi_{0}\right)\right\|$ as follows:

$$
\left\|\grave{B}\left(\varphi_{0}\right)^{-1} B\left(\varphi_{0}\right)\right\| \leq \alpha_{0}\left\|B\left(\varphi_{0}\right)\right\| \leq \mu_{0},
$$

where

$$
\mu_{0}=\alpha_{0}\left(\left\|\varphi_{0}\right\|+|\lambda| c_{0}(r)(1+\|v\|)+\|G(t, 0)\|_{c}\right),
$$

Since

$$
a=\left\|\grave{B}\left(\varphi_{0}\right)^{-1} B\left(\varphi_{0}\right)\right\|,
$$

hence

$$
a \leq b\left[\left\|\varphi_{0}\right\|+|\lambda| c_{0}(r)(1+\|v\|)+\|G(t, 0)\|_{c}\right],
$$

and

$$
b \leq \alpha_{0}
$$

therefore, the following theorem is valid. 
Theorem 4.2 Suppose that the equation (2.3)has a unique positive root $r_{*}$ in $[0, R]$ and $\psi(R) \leq 0$. Then the equation $B(\varphi)=0$ has a unique solution $\varphi_{*}$ in $S\left(\varphi_{0}, R\right)$ and the NewtonKantorovich approximations:

$$
\varphi_{n}=\varphi_{n-1}-\grave{B}\left(\varphi_{n-1}\right)^{-1} B\left(\varphi_{n-1}\right), \quad n \in N,
$$

are defined for all $n \in N$, belong to $S\left(\varphi_{0}, r_{*}\right)$ and converges to $\varphi_{*}$. Moreover, the following estimate holds

$$
\left\|\varphi_{n+1}-\varphi_{n}\right\| \leq r_{n+1}-r_{n}, \quad\left\|\varphi_{*}-\varphi_{n}\right\| \leq r_{*}-r_{n},
$$

where the sequence $\left(r_{n}\right)_{n \in N}$ converges to $r_{*}$, is defined by the recurrence formula

$$
r_{0}=0, \quad r_{n+1}=r_{n}-\frac{\psi\left(r_{n}\right)}{\psi\left(r_{n}\right)}, \quad n \in N
$$

We will illustrate the theorem 4.2 by the following example. Consider the nonlinear function

$$
f(u)=\frac{1}{6} u^{3}+\frac{1}{6} u^{2}-\frac{5}{6} u+\frac{1}{3}
$$

with derivative

$$
\grave{f}(u)=\frac{1}{2} u^{2}+\frac{1}{3} u-\frac{5}{6}
$$

it's clear that

$$
\begin{aligned}
\frac{\left\|\grave{f}\left(u_{1}\right)-\grave{f}\left(u_{2}\right)\right\|}{\left\|u_{1}-u_{2}\right\|} & \leq \frac{1}{6}\left[\left\|3\left(u_{1}+u_{2}\right)\right\|+2\right] \\
& \leq r+\frac{1}{3},
\end{aligned}
$$

therefore we get

$$
k(r)=r+\frac{1}{3}
$$

Obviously, the scaler equation (2.3) takes the form

$$
\psi(r)=a+\frac{b}{6} r^{3}+\frac{b}{6} r^{2}-r .
$$

The equation

$$
\psi(r)=0,
$$

has a unique positive solution $r_{*}$ in $[0, R]$ if and only if

$$
\left[\frac{q}{2}\right]^{2}+\left[\frac{p}{3}\right]^{3}>0
$$

where ,

$$
p=-\frac{1}{3}-\frac{6}{b} \quad \text { and } \quad q=\frac{2}{27}+\frac{2}{b}+\frac{6 a}{b} .
$$

Hence, the function $f(u)=0$ has a unique solution $u_{*}$ in $S(0, R)$ and the assumptions of theorem (4.2) are verified.

Received: October 2008. Revised: February 2009. 


\section{References}

[1] N. U. Ahmed, Semigroup Theory with Applications to Systems and Control, Pitman Research Notes in Mathematics Series, 246. Longman Scientific \& Technical, Harlow; John Wiley \& Sons, New York, 1991.

[2] N. U. Ahmed, Dynamic Systems and Control with Applications, World Scientific Publishing Co. Pte. Ltd., Hackensack, NJ, 2006.

[3] N. U. Ahmed, Systems governed by impulsive differential inclusions on Hilbert spaces, Nonlinear Anal. 45 (2001), 693-706.

[4] S.M. Amer And S. Dardery, On the theory of the of nonlinear singular integral equations with shift in Hölder spaces, Forum Math.17(2005),753-780.

[5] S.M. Amer ANd A.S. NAgdy, On the modified Newton's approximation method for the solution of nonlinear singular integral equations, Hokkaido Mthematical Journal 29(2000)59-72.

[6] S.M. Amer, On the approximate solution of nonlinear singular integral equations with positive index, Int.J.Math.Math.Sci,19(2)(1996)389-396.

[7] J. W. Appel, E. De Pascale, N. A. Eevkhuta and P. P. Zabrejko, On the two-step Newton method for the solution of nonlinear operator equations, Math.Nachr.172(1995)5-14.

[8] Argyro, Ionnnis K., Othe convergence of Newton's method for a class of nonsmooth operators; Journal of Computational and Applied Mathematics 205(2007)584-593.

[9] Espedito de Pascale-PJptr P.Zabrejko, New convergence criteria for the Newton-Kantorovich method nd some applications to nonlinear integral equations, REND.SEM.MAT.UNIV.PADOV A, vol-100(1998)

[10] Filomena Cianciaruo and Espedito De Pascale, Estimates of majorizing sequences in the Newton-Kantorovich method :Afurther improvement, J.Math.Anal.Appl.322(2006)329-335.

[11] F.D. Gakhov, Boundary Value Problems, Dover Publ .N.Y.,1990.

[12] A.I. Guseinov and Mukhtarov KH . Sh., Introduction to the Theory of Non-linear Singular Integral Equations, (In Russian), Nauk . Moscow,1980.

[13] D. Jinyuan, The collocation methods and singular integral equations with Cauchy kernel, Acta Math.Sci.20(B3)(2000)289-302.

[14] L.V. Kantorovich and G.P Akilov, Functional Analysis, Pergamon Press.Oxford.1982.

[15] E.G. Ladopoulos And V.A. Zisis, Non-linear singular integral approximations in Banach spaces, Non-linear Analysis,Theory,Methods and Applications,Vol. 26, No.7, (1996)1293-1299.

[16] S.G. Mikhlin And S. Prossdorf, Singular Integral Operator, Akademie- Verlag,Berlin,1986.

[17] N.I. Muskhelishvill, Singular Integral Equations, Englih Trans I.;Noordhoff Ltd.Groningen 1968. 
[18] A. Pedas and G. Vainikko, Supper convergence of piecwise polyn-omial colloctions for nonlinear weakly singular integral equtions, Journal of integral equations and applications vol 9, no.4,(1997)379-406.

[19] W. Pogorzelski, Integral Equations and Their Applications, vol 1, Oxford Pergamon Press and Warszawa, PWN,1966.

[20] Qingbiao Wu and Yueqing Zhao, Newton-Kantorovich convergence theorem for the inversefree Jarratt method in Banach space, Applied Mathematics and Computation;volume 179,issue 1, 1 August 2006, pages 39-46.

[21] Qingbiao Wu And Yueqing ZhaO, Third-order convergence theorem by using majorizing function for a modified Newton method in Banch space, Applied Mathematics and Computation;175(2006)1515-1524.

[22] M.H Saleh and S.M Amer, On the mechanical qudrature method for solving singular integral equations with Hilbert kernel, Journal of concrete and Applicable Mathematics vol.6,No.4(2008)387402.

[23] Subhra.Bhattachrya and B.N. Maudel, Numerical solution of a singular integro-differential equations, Applied Mathematics and Computation;195(2008)346-350. 